\title{
Environmental Effects on Galaxy Evolution: Multifrequency study of nearby clusters
}

\author{
H. Bravo-Alfaro ${ }^{1,2}$ Y. Venkatapathy, ${ }^{1}$ F. Durret, ${ }^{2}$ C. A. Caretta $^{1}$ and \\ V. Gamez ${ }^{1}$ \\ ${ }^{1}$ Departamento de Astronomia, Universidad de Guanajuato \\ Apdo. Postal 144, Mexico. email: hector@astro.ugto.mx \\ ${ }^{2}$ Institut d'Astrophysique de Paris, \\ UPMC-CNRS, UMR7095, 98bis Bd Arago, 75014, Paris, France
}

\begin{abstract}
We show first results of a multifrequency survey focused on the mechanisms driving the transformation of cluster galaxies, from late to earlier S0-types. We compare the ram-pressure effects seen on $21 \mathrm{~cm}-\mathrm{HI}$ images with those mechanisms affecting the old stellar disks seen on deep optical and NIR images. Our targets are the Abell clusters A85, A496, A2670, which span different relaxation degrees, masses and X-ray luminosities.
\end{abstract}

Keywords. galaxies: evolution — galaxies: clusters: individual: (Abell 85, 496, 2670)

\section{Results}

The striking absence of spirals near the cluster cores is accompanied by the opposite growing population of lenticulars in such high density regions (the density-morphology relation.) In general it is accepted that environment plays and important role in this matter, but determining which are the responsible physical processes is still a matter of debate. Such mechanisms are classified in two types, hydrodynamic (e.g. ram pressure stripping) and gravitational ones (e.g. galaxy-galaxy and galaxy-cluster interactions.)

With the aim to study both types of physical mechanisms, we obtained a set of $21 \mathrm{~cm}$ HI (NRAO-VLA) and deep optical-NIR images (2m-SPM, 3.6m-CFHT) covering large volumes of the Abell clusters: A85, A496 and A2670. They span the redshift range, 0.033 - 0.066, and were chosen having different $L_{X}$ and relaxation degrees. One of our goals is trying to correlate such cluster properties with those shown by individual infalling galaxies. Follows a summary of our main results so far (see Bravo-Alfaro et al., in prep.):

- Abell 85: This is a well known X-ray bright cluster $\left(9.4 \times 10^{44} \mathrm{erg} / \mathrm{s} \mathrm{cm}^{2}\right)$ being part of a supercluster having other ten members. A 85 has a number of substructures and our HI-survey shows a rather deficient cluster with only 10 HI-detections.

- Abell 496: This is an isolated system, not being part of any supercluster, and shows a clear massive group infalling from the NW. Our VLA-HI imaging unveils a rich gas cluster having above $50 \mathrm{HI}$-detections.

- Abell 2670: This is an isolated and very young (dynamically) cluster. The expected high number of HI-detections (around 70) are projected along a NE-SW axis. A preliminary study of some individual cases already shows remarkable cases of gas disruption, clear offsets between the HI and optical disks, and strong gas deficiencies. Furthermore, some substructures are detected by using the 3-D optical galaxy distribution. 